\title{
Temporal Control of Axonal Transport: The Extreme Case of Organismal Ageing
}

\author{
Francesca Mattedi and Alessio Vagnoni*
}

Department of Basic and Clinical Neuroscience, Maurice Wohl Clinical Neuroscience Institute, IoPPN, King's College London, London, United Kingdom

A fundamental question in cell biology is how cellular components are delivered to their destination with spatial and temporal precision within the crowded cytoplasmic environment. The long processes of neurons represent a significant spatial challenge and make these cells particularly dependent on mechanisms for long-range cytoskeletal transport of proteins, RNA and organelles. Although many studies have substantiated a role for defective transport of axonal cargoes in the pathogenesis of neurodevelopmental and neurodegenerative diseases, remarkably little is known about how transport is regulated throughout ageing. The scale of the challenge posed by ageing is considerable because, in this case, the temporal regulation of transport is ultimately dictated by the

OPEN ACCESS

Edited by:

Shermali Gunawardena, University at Buffalo, United States

Reviewed by:

Majid Hafezparast,

University of Sussex, United Kingdom

Angels Almenar,

University of California, San Diego, United States

Victor Faundez,

Emory University, United States

*Correspondence:

Alessio Vagnon

alessio.vagnoni@kcl.ac.uk

Specialty section: This article was submitted to

Cellular Neuropathology,

a section of the journa

Frontiers in Cellular Neuroscience

Received: 31 May 2019

Accepted: 09 August 2019

Published: 23 August 2019

Citation:

Mattedi F and Vagnoni A (2019) Temporal Control of Axonal Transport:

The Extreme Case of Organismal Ageing. Front. Cell. Neurosci. 13:393. doi: 10.3389/fncel.2019.00393 length of organismal lifespan, which can extend to days, years or decades. Recent methodological advances to study live axonal transport during ageing in situ have provided new tools to scratch beneath the surface of this complex problem and revealed that age-dependent decline in the transport of mitochondria is a common feature across different neuronal populations of several model organisms. In certain instances, the molecular pathways that affect transport in ageing animals have begun to emerge. However, the functional implications of these observations are still not fully understood. Whether transport decline is a significant determinant of neuronal ageing or a mere consequence of decreased cellular fitness remains an open question. In this review, we discuss the latest developments in axonal trafficking in the ageing nervous system, along with the early studies that inaugurated this new area of research. We explore the possibility that the interplay between mitochondrial function and motility represents a crucial driver of ageing in neurons and put forward the hypothesis that declining axonal transport may be legitimately considered a hallmark of neuronal ageing.

Keywords: axonal transport, ageing, neurons, mitochondria, in vivo imaging, intracellular trafficking, model organisms

\section{INTRODUCTION}

Ageing is a process characterised by progressive decline of cellular and organismal functions. How neurons age is a fascinating biological question with vast societal implications, not least due to the ever-increasing life expectancy, but also because ageing is the major risk factor for dementia and other disorders of the nervous system (Niccoli and Partridge, 2012). Neuronal cells heavily rely on mechanisms for the delivery of essential intracellular cargoes within axons and dendrites; for example, mobilisation of mitochondria to regions of high energy demand, trafficking of 


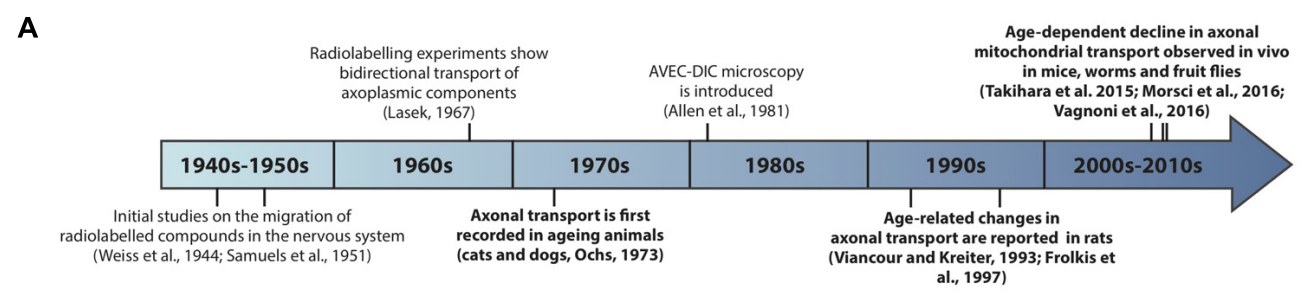

B

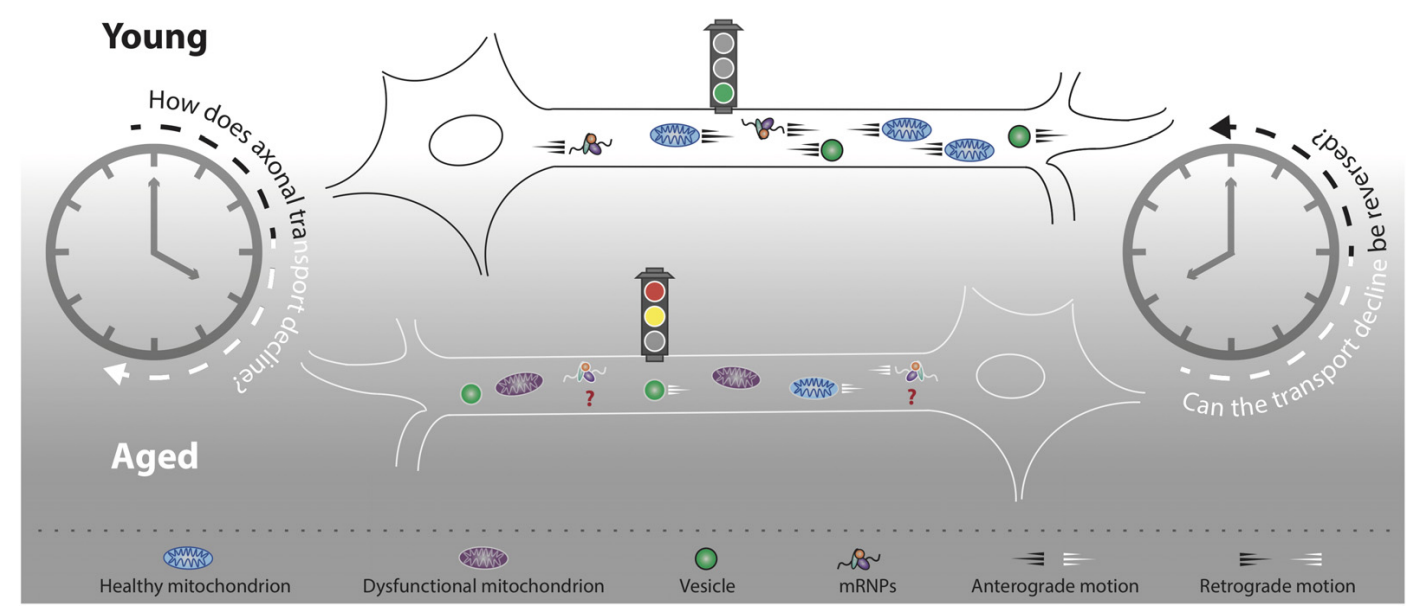

FIGURE 1 | Decline of axonal transport is a hallmark of neuronal ageing. (A) Timeline of selected milestones in the study of axonal transport during ageing. In bold, studies of particular interest for the scope of this review. (B) Decline of axonal cargo transport during ageing is a common feature across different neuronal populations of several model organisms, although the precise mechanisms of transport decline are not well understood. Not all cargo transport equally declines during ageing (refer to main text for further details) and the motility of many essential cargoes, for instance mRNPs, has not yet been investigated (red question marks). In addition to transport decline, cargoes such as mitochondria undergo an age-dependent functional decay (purple mitochondria). Transport decline correlates with decreased cellular function: can the decline in cargo motility be reversed in an attempt to improve neuronal health during ageing?

mRNA and ribosomal subunits for local translation, signalling endosome-mediated delivery of survival factors and autophagosome-mediated clearance of damaged organelles. As the cellular equivalent of a conveyor belt, when the transport process is interrupted, the functional implications for the neurons are profound. It is therefore not surprising that mutations in genes encoding axonal transport machinery are linked to human disease and that axonal transport impairments have been implicated in the pathogenesis of many neurological diseases (De Vos et al., 2008; Millecamps and Julien, 2013). However, whether this process plays a role in the ageing of neurons has always remained elusive, mainly due to a lack of suitable models to study transport during organismal ageing. Initial studies using radiolabelled amino acids to track axonal transport in neurons of ageing cats and dogs effectively inaugurated a new area of research (Ochs, 1973; Figure 1A). A number of technical advances have recently provided new opportunities to study neuronal trafficking during ageing in different model organisms (Sleigh et al., 2017), thus injecting new life into a largely unexplored research field. In this review, we provide a summary of the current knowledge of intracellular trafficking in ageing neurons, highlighting pressing research questions and new opportunities presented by the latest technical developments.

\section{INTRACELLULAR TRAFFICKING DURING NEURONAL AGEING - A BRIEF HISTORICAL PERSPECTIVE}

Life implies movement and the concept of motion has been essential for our understanding of the physical world since ancient times (Hall, 1965; Kosman, 1969). It was not, however, until the invention of the first single-lensed microscopes that the motion of the microscopic world began to capture the imagination of scientists (Lane, 2015). Further technical advancement, for instance the invention of microscopes with achromatic lenses, started a new era of scientific discoveries that, throughout the $19^{\text {th }}$ century, saw scientists striving to characterise the 'living jelly' (gelée vivant, Dujardin, 1835), or 'living protoplasm' (Huxley, 1869). The protoplasm, which we now know to be the cytoplasm, was thought to carry all the vital cellular functions and therefore constitute the physical basis of life (Huxley, 1869). Fast forward to the years 19501970 to see the first studies carried out in the axons of neurons describing the distribution and migration of cytoplasmic components by classic tracing methods using radiolabelled isotopes (Weiss et al., 1945; Samuels et al., 1951; Lubinska, 1964; Lasek, 1967; Weiss, 1967; Figure 1A). Mammalian nerves, 
and especially the sciatic nerve, were particularly suitable for this purpose as they are long, contain many axons and can be used to recover abundant radioactive extract to indirectly quantify the progression of the axoplasmic flow. These initial studies paved the way for further work aimed at characterising the transport of cellular components in the nervous system of ageing animals (Ochs, 1973). Ten years later, a monitor was attached to a microscope and AVEC-DIC (video-enhanced contrast, differential interference contrast) microscopy was born (Allen et al., 1981), which provided the first direct, real-time evidence of 'orthograde' (i.e., toward the cell periphery) and retrograde (i.e., toward the cell soma) organelle movements in the giant axon of the squid (Allen et al., 1982; Brady et al., 1982).

Over the course of the next two decades, many observations were made suggesting that the movement of specific cargoes within axons may be reduced with age in rodent neurons (Geinisman et al., 1977; Stromska and Ochs, 1982; Brunetti et al., 1987; McQuarrie et al., 1989; Castel et al., 1994; Frolkis et al., 1997; Uchida et al., 2001; Li et al., 2004). These early studies relied mostly on radiolabelling techniques, at times combined with nerve ligation assays (Fernandez and Hodges-Savola, 1994) or the emerging AVEC-DIC microscopy (Viancour and Kreiter, 1993), and effectively opened up a new avenue of research: the study of intracellular trafficking in the nervous system of ageing animals (Figures 1A,B). Because the displacement rates of the axoplasmic flow were generally calculated indirectly by biochemical analyses of the dissected tissue, the spatial and temporal resolution of these experiments was inevitably limited. Distinguishing between cargo-specific and general decline of transport throughout ageing was therefore often challenging. It was only with the discovery of fluorescent proteins, more recently coupled with the development of real-time in vivo imaging assays, that a clearer picture has begun to emerge on how neuronal trafficking is orchestrated in ageing organisms.

\section{MODEL ORGANISMS: ONE MODEL DOES NOT FIT ALL}

A major bottleneck for ageing studies of neuronal cargo transport has been the lack of suitable models to study this process in real-time during the lifespan of an animal. Substantial progress has recently been made using novel assays in several model organisms, which we briefly review in this paragraph.

\section{Mus musculus ('The Mouse') Mean Lifespan of Laboratory Mice: 2 Years (Flurkey et al., 2007)}

The study of neuronal trafficking in its natural habitat (Lichtman and Fraser, 2001) was significantly boosted by a body of work aimed at clarifying the dynamics of mitochondrial movement in the peripheral nerves of the MitoMouse. This transgenic mouse model, in which fluorescent proteins are specifically targeted to the matrix of the neuronal mitochondria, showed that the mitochondria maintain their high dynamicity in adult animals in vivo (Misgeld et al., 2007).
More recent studies with the MitoMouse have showed that the axonal transport of mitochondria undergoes an age-dependent decline in both retinal ganglion cells (i.e., the CNS) in situ (Takihara et al., 2015) and tissue explants of the sciatic nerve (i.e., the PNS) (Gilley et al., 2012; Milde et al., 2015). While the number of motile mitochondria is not overall affected in the mouse CNS up to 25 months of age, their dynamic properties (e.g., duration of movement and distance travelled) progressively decline as the animals age (Takihara et al., 2015). In the PNS ex vivo, the fraction of motile mitochondria starts declining already at 6 months of age. This is mirrored by an age-dependent reduction in the transport of the Golgi-derived NMNAT2 vesicles from 3 to 6 months of age in both CNS and PNS explants (Milde et al., 2015). With the exception of reduced vesicular velocity in the PNS tissue explants (Milde et al., 2015), cargo speeds remain largely unaffected by ageing in the mouse nervous system both in situ and ex vivo. Interestingly, ageing exacerbates the mitochondrial transport defects in tissue explants of a mouse model of tauopathy (Gilley et al., 2012), possibly due to alteration of microtubule dynamics, and in a mouse model of glaucoma in vivo (Takihara et al., 2015).

Age-dependent decrease of mitochondrial transport was also observed in dorsal root ganglia neurons (DRGs) cultured from 5-month old mice overexpressing tau P301S, compared to DRGs cultured from younger mice of the same genotype (Mellone et al., 2013). Recent work showed that the biogenesis of the autophagosome decreases in cultured DRGs from aged mice (Stavoe et al., 2019). Combined with evidence that hippocampal neurons cultured from ageing animals display mitochondrial functional decline (Parihar and Brewer, 2007), these findings indicate that in vitro cultures of neurons obtained from ageing animals could be used to recapitulate significant aspects of neuronal ageing and complement the in vivo work.

In spite of the age-dependent decline in cargo transport observed in different neuronal populations, transport reduction does not seem to be a general feature of neuronal ageing in mice in vivo. By performing intravital imaging from the mouse sciatic nerve (Bolea et al., 2014; Gibbs et al., 2016), Sleigh and co-authors showed that trafficking of neurotrophin-containing signalling endosomes is not affected in animals up to 18 months of age (Sleigh and Schiavo, 2016; Sleigh et al., 2018). Signalling endosomes mainly rely on mechanisms for retrograde transport (Schmieg et al., 2014) as opposed to the bidirectional motility of Golgi-derived NMNAT2 vesicles (Milde et al., 2015). The different dynamics of these vesicular cargoes during ageing may therefore reflect inherently distinct dynamic properties. Alternatively, the discrepancy might be due to physiological differences between the ex vivo and in vivo systems that would impact on specific subset of cargoes. Whatever the case, these findings highlight the need of more work to precisely dissect the dynamics of cargo transport during ageing.

The availability of new techniques to image neuronal cargo transport in vivo and ex vivo has opened up the possibility to study how intracellular trafficking is regulated in space and time in a more physiological setting relevant for ageing research. These studies so far have mainly focused on the mitochondria and led to refine our understanding of the relationship between 
mitochondrial dynamics and other aspects of cellular physiology, from neuronal activity (Sajic et al., 2013; Faits et al., 2016) to cellular redox state (Breckwoldt et al., 2014) and disease contexts (Bilsland et al., 2010; Sorbara et al., 2014). Although the mouse experiments have demonstrated the significance of studying transport within an ageing nervous system, this type of study is technically challenging and time consuming. In addition, the cost attached to it is prohibitive for many laboratories and the techniques used remain largely invasive (i.e., dissection could significantly interfere with the physiology of the processes observed). More recently, new work revealed the suitability of the fruit fly Drosophila melanogaster and the nematode Caenorhabditis elegans to study axonal transport in ageing animals. Due to the shorter lifespan of the invertebrate models, a clear advantage of using these systems is the possibility of making discoveries more rapidly. Key findings obtained in fruit flies and worms could then be validated in mammalian neurons to determine the evolutionary conservation and wider significance of such discoveries.

\section{Drosophila melanogaster ('The Fruit Fly') Mean Lifespan of Laboratory Fruit Flies: $~ 50$ Days (Linford et al., 2013)}

The fruit fly is a prime model organism for ageing studies and it has led to seminal discoveries in the ageing field (Clancy et al., 2001; Bjedov et al., 2010). Drosophila has been extensively used for studies of axonal transport during larval and pupal developmental stage, providing invaluable insight into the mechanisms regulating neuronal trafficking (Gunawardena and Goldstein, 2001; Guo et al., 2005; Pilling et al., 2006; Wang and Schwarz, 2009; Pathak et al., 2010; Xiong et al., 2010; Avery et al., 2012; Reis et al., 2012; Quraishe et al., 2013; Medioni et al., 2015; Baldwin et al., 2016; Dey et al., 2017). A new assay that exploits the transparent wing of Drosophila has recently allowed studying this process in a subset of peripheral neurons of adult animals, with the advantage of monitoring cargo transport as the animals age (Vagnoni and Bullock, 2016). This approach, in which the neurons are imaged from live animals without surgical exposure of the nerve tracts, revealed that the fraction of transported mitochondria, but not their velocity or run length, declines early during ageing (Vagnoni et al., 2016). Halting mitochondrial transport specifically in adult neurons accelerates the appearance of protein aggregation, highlighting the relevance of mitochondrial trafficking for neuronal homeostasis. In this system, the transport of dense core vesicles is largely unaffected during ageing (Vagnoni et al., 2016; Vagnoni and Bullock, 2018). Therefore, as in the mouse sciatic nerve, early decline in axonal transport is not a general feature of ageing, ruling out major disruption to the cytoskeletal network, at least at an early stage. This work also showed that aged neurons are still capable of reversing a prolonged transport decline with an acute intervention in later life by boosting the cAMP/PKA pathway (Vagnoni and Bullock, 2018).

The short lifespan of Drosophila makes ageing studies inevitably less challenging than in murine models. Combined with the power of fly genetics, this system warrants fast progress in understanding the mechanisms regulating axonal transport during ageing and the relationship between transport and neuronal function during the animal lifetime. It would be informative to expand these studies to the CNS of flies, for instance by using ex vivo explants of adult brains (Ayaz et al., 2008) or the dissected ventral nerve cord (Kudumala et al., 2017), to understand whether decline in mitochondrial transport is shared between different neuronal types.

\section{Caenorhabditis elegans ('The Worm') Mean Lifespan of Laboratory Worms: $\sim 17$ Days (Gems and Riddle, 2000)}

Since the first DNA mutations correlating with lifespan extension were discovered in C. elegans (Klass et al., 1983), a string of discoveries were made in this model organism that challenged the classical view of evolutionary biologists that single mutations could not have a strong effect on lifespan (Kenyon et al., 1993; Morris et al., 1996). As with the fruit fly, the genetic tractability of this organism, coupled with its even shorter lifespan, makes it an excellent model for ageing studies of organelle transport.

Microfluidic devices have been developed for imaging neuronal transport in live worms throughout development and adulthood. By exploiting the translucent nature of these animals, robust transport of Rab3-positive synaptic vesicles can be readily observed in single sensory axons (Mondal et al., 2011). With ageing, the fraction and speed of the vesicles moving in the anterograde direction is decreased in D9 motor neurons and it correlates with impaired synaptic transmission (Li et al., 2016). Mitochondrial transport declines with age in mechanosensory neurons and this is associated with reduced mitochondrial density in the axons and a decreased ability of the animals to respond to oxidative stress throughout ageing (Morsci et al., 2016). Interestingly, by analysing transport in long-lived daf-2 and eat-2 mutant worms, the authors show that the prolonged maintenance of mitochondria trafficking is associated with the lifespan extension typical of these animal models and with increased mitochondrial resistance to oxidative stress, thus implicating the insulin-like signalling pathway in the regulation of mitochondrial motility and function during ageing. Redox imbalance has been shown to affect mitochondrial distribution in cultured mammalian cells (Debattisti et al., 2017) and in Drosophila adult neurons in vivo (Smith et al., 2019). It would be interesting to test whether modulation of cellular stress directly influences mitochondrial transport in this context. Mitochondrial motility is also affected by injury (Rawson et al., 2014) and in a C. elegans model of tauopathy (Fatouros et al., 2012), although it is not known whether ageing has any effect on the phenotypes observed.

\section{Danio rerio ('The Fish') \\ Mean Lifespan of Laboratory Fish: 3.5 Years (Gerhard et al., 2002)}

Danio rerio, aka zebrafish, has emerged as an important model for studying neuronal function in an organismal context (Walker et al., 2013; Patten et al., 2014), and recent developments have provided powerful assays to measure the transport of 
mitochondria in a subset of CNS and PNS nerves during development (Plucinska et al., 2012; O’Donnell et al., 2013; Bergamin et al., 2016; Xu et al., 2017; Mandal et al., 2018). These studies have shed light on the distribution of mitochondria within the complex architecture of a neuron in situ and have expanded our understanding of the consequences of diseasecausing mutations on transport. As with adult fruit flies and worms, the accessibility of zebrafish neurons to microscopy observation is made possible by the translucency of the animal cuticle. This advantage is, however, restricted to developmental stages, limiting ageing studies of organelle transport. Being able to perform live imaging in adult tissues would represent an exciting advancement, for instance by exploiting the see-through nature of the medaka fish (Wakamatsu et al., 2001). The possibility of combining in situ imaging of organelle transport with shortlived fish models used in ageing research (Ding et al., 2010; Harel et al., 2015) could have a major impact and provide novel opportunities for the field.

\section{Homo sapiens ('The Humans') Mean Life Expectancy of Humans (2015-2020): 72 Years (UN DESA, 2017)}

The development of new methods in a number of animal models has begun to clarify fundamental mechanisms of cargo transport in ageing neurons and, more broadly, has added to our understanding of the cell biology of the ageing nervous system. It is not straightforward, however, to translate fundamental discoveries attained in model organisms to the ageing process of humans for the benefit of human health. Being able to study this process in a human model of neuronal ageing would help to address this knowledge gap in the field. Human-induced pluripotent stem cells (hiPSC) offer great potential to study intracellular trafficking in neurons derived from patients affected by a range of neurodegenerative diseases. Features associated with cellular age can be induced in these neurons, either by directly interfering with ageing markers or after an extended period in culture of several months (Liu et al., 2011; Ziff and Patani, 2019). Recent developments in stem cell technology have provided novel tools based on cultures of neurons derived directly from fibroblasts of human donors (iNeurons) (Mertens et al., 2015). Unlike hiPSC, these cells are not reprogrammed to an embryonic state. Thus, iNeurons derived from young and old individuals display age-specific transcriptional signatures, along with markers of mitochondrial ageing (Kim et al., 2018), suggesting they are suitable for comparisons of young and old cellular states. A notable advantage of the in vitro cellular system is the possibility of carrying out in-depth mechanistic studies that would be more challenging in vivo.

Although the fundamental biological insights obtained in model systems in vitro and in vivo are crucial to advance the field, it is desirable to validate these findings in what arguably remains the best in vivo model, the humans themselves. Imaging approaches using manganese-enhanced magnetic resonance imaging $\left(\mathrm{Mn}^{2+}-\mathrm{MRI}\right)$ have revealed a decrease of the bulk transport in the olfactory system of aged mice (Cross et al., 2008). This non-invasive approach could potentially be translated to humans, with the possibility of recording transport rates in longitudinal studies from living human brains. These advantages, however, need to be weighed against the potential toxicity of $\mathrm{Mn}^{2+}$ and the inevitable lack of tracer specificity which only reports on the bulk axoplasmic flow. Alternatively, pulse doses of non-radioactive tracers, such as heavy water $\left({ }^{2} \mathrm{H}_{2} \mathrm{O}\right)$, could be followed by cerebrospinal fluid (CSF) sampling in young and old individuals through lumbar puncture and mass spectrometric analysis of the labelled proteins (Fanara et al., 2012). CSFbased biomarkers of neuronal cargo transport could then be identified to monitor defective transport during ageing. More recently, an exciting approach combining adaptive optics and optical coherence tomography (AO-OCT) has been applied to imaging fine neuronal details in the human retina in vivo (Liu et al., 2017). Enhanced visualisation of single axonal fibres (Gray et al., 2008), combined with the topical application of nontoxic chemical markers of subcellular structures, would open up the unprecedented opportunity to peer into the intracellular dynamics of the ageing nervous system of humans.

\section{MITOCHONDRIA, AN ATYPICAL (BUT ESSENTIAL) CARGO}

Mitochondria have a central role in governing energy metabolism via ATP production and are regarded as the 'powerhouse of the cell' (Siekevitz, 1957). Their fundamental role extends to numerous other processes essential for life, including fatty acid beta-oxidation, calcium signalling and steroid synthesis, and mitochondrial dysfunction is widely regarded as one of the hallmarks of ageing (Bishop et al., 2010; López-Otín et al., 2013; DiLoreto and Murphy, 2015).

Many studies have focused on the mechanisms regulating mitochondrial motility and showed that disruption of mitochondrial transport invariably results in altered neuronal function (Sheng and Cai, 2012). However, mitochondria are atypical axonal transport cargoes as most of them can remain stationary at any given time (Hollenbeck and Saxton, 2005; Gutnick et al., 2019). Despite their lack of motility relative to other cargoes, reduction in mitochondrial movements is linked to decreased cellular health during ageing and disease, and declining transport appears to be an evolutionary conserved hallmark of ageing across several neuronal subtypes, from invertebrates to mammals (Takihara et al., 2015; Morsci et al., 2016; Vagnoni et al., 2016). On the other hand, upregulation of mitochondrial transport, for instance by boosting the cAMPPKA pathway, correlates with increased cellular health in aged Drosophila neurons (Vagnoni and Bullock, 2018) and underlies synapse formation in Aplysia neurons (Badal et al., 2019). In fruit flies and worms, upregulation of mitochondrial transport also correlates with the suppression of Wallerian degeneration (Avery et al., 2012) and is protective against axotomy-triggered degeneration (Rawson et al., 2014). It has also been shown that transport upregulation in vitro is necessary for the maintenance of neuronal homeostasis after injury (Zhou et al., 2016) and is sustained after regeneration in young and old mice ex vivo (Mar et al., 2014; Milde et al., 2015). It would be interesting to 
expand these studies in ageing animals in vivo, for instance by exploiting current assays for imaging the mouse spinal cord after injury (Kerschensteiner et al., 2005; Davalos et al., 2008; Farrar et al., 2012). Increasing mitochondrial trafficking may not, however, always represent a viable option for improving cellular health (Zhu and Sheng, 2011; Faits et al., 2016) suggesting context-specific functional outcome of transport upregulation.

\section{HOW IS MITOCHONDRIAL DISTRIBUTION MAINTAINED THROUGHOUT AGEING? THE PROBLEM OF 'TRANSPORT IMBALANCE'}

The advent of systems for in vivo imaging of organelle transport have, on one hand, provided new platforms to explore open questions in the field and, at the same time, presented us with new challenging questions (Table 1). On the other hand, these new methodologies have validated the general principles governing neuronal trafficking discovered in cultured neurons and expanded them to an in vivo setting. There are striking similarities in the properties of mitochondrial transport between in vitro and in vivo systems. For instance, more organelles are consistently transported in the anterograde than in the retrograde direction, both in young and old neurons (Vagnoni et al., 2016; Misgeld and Schwarz, 2017). What happens to the mitochondria that, seemingly, do not make the trip back along the axon is an open question and whether interfering with the anterograde/retrograde ratio has an impact on ageing is unknown. It is still unclear if the 'transport imbalance' may be coupled to the recycling of the organelles (Miller and Sheetz, 2004; Maday and Holzbaur, 2014; Lin et al., 2017) with in vitro studies suggesting that motile mitochondria may become anchored at sites of functional requirement such as synapses or nodes of Ranvier (Chang et al., 2006; Obashi and Okabe, 2013; Lewis et al., 2016; Gutnick et al., 2019). A notable exception are the ALM sensory neurons of C. elegans where mitochondria are transported with a retrograde bias throughout life (Morsci et al., 2016). Interestingly, these neurons lack synaptic connections, suggesting that synaptic activity may play a crucial role in determining the anterograde transport bias of mitochondria in mature neurons. Whatever the mechanism accounting for the majority of mitochondria constantly travelling in the anterograde direction, this is an evolutionary conserved feature likely to support a fundamental cellular demand.

Imbalanced mitochondrial biogenesis (López-Lluch et al., 2008; Seo et al., 2010) and changes in mitochondrial fission and fusion (Cao et al., 2017) were observed during ageing, although a mechanistic association with mitochondrial transport and distribution is still unclear at this stage. It is not known whether mitochondrial anterograde bias throughout life and age-dependent transport decline lead to toxic accumulation in the soma or to a depletion of the organelle over time (Morsci et al., 2016). This is an open question which deserves further investigation.

A progressive and specific decline in the motility of mitochondria has been observed during developmental maturation of cortical axons in live mice up to 45 days of age (Lewis et al., 2016; Smit-rigter et al., 2016) and in maturing dendrites of ganglion cells of retinal explants (Faits et al., 2016). The general reduction in motility is, in these cases, necessary to distribute the organelles by stably anchoring them at specific cellular sites. In this light, it is tempting to speculate that decline in mitochondrial transport in mature neurons may be part of a run-on of developmental processes (Azpurua et al., 2018; Ezcurra et al., 2018). Thus, early transport decline, beneficial during development, may become detrimental in later life when the functional demand of a neuron changes, by contributing to age-dependent decline of neuronal functions such as reduction of ATP production (Kim et al., 2018), impaired calcium homeostasis (Nikoletopoulou and Tavernarakis, 2012) and increased oxidative stress (Castelli et al., 2019). Choosing the appropriate window of time to upregulate the transport therefore appears crucial to be able to meaningfully impact on neuronal health in later life (Vagnoni and Bullock, 2018).

\section{OUTLOOK}

In spite of the strong association between axonal transport and the maintenance of neuronal health, it is unclear how modulation

TABLE 1 | Outstanding and new questions facing the field of neuronal trafficking during ageing.

Outstanding What are the functional consequences of transport decline of specific cargoes?

Can we tease out cell-autonomous from non-cell-autonomous mechanisms affecting cargo transport?

Is the cytoskeleton structure rearranged during ageing? If so, how does this impact transport?

Do specific post-translational modifications of key components of the transport machinery (e.g., motors, adaptors, microtubules, microtubule-associated proteins, and regulatory kinases) affect cargo trafficking during ageing?

How does a neuron sustain the transport imbalance of certain cargoes (i.e., many more cargoes continously transported with an anterograde bias)?

New What is the functional relevance of the timing of cargo decline during ageing (i.e., early vs. late decline)?

Are specific subsets of neurons more sensitive to age-dependent transport decline that could predispose them to degeneration, hence contributing to neuronal specificity in some neurodegenerative conditions?

Are neurons able to sustain a prolonged upregulation of cargo transport, either through genetic or chemical modification, that could alleviate age-related dysfunction?

What is the composition of the cytoplasmic milieu in ageing cells and how does this affect cargo transport?

Does the architecture and recruitment of the cargo transport machinery change during ageing? Does the changing abundance of motor and adaptor proteins favour the formation of specific transport complexes that are less likely to form in early life? 


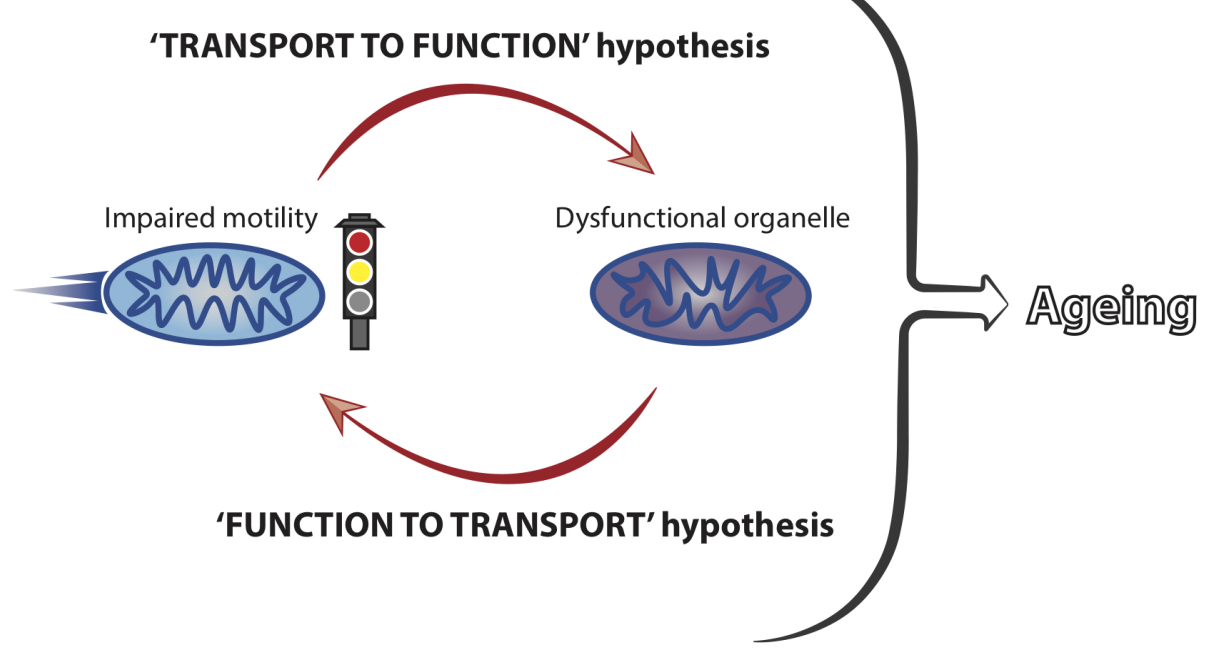

FIGURE 2 | Mitochondrial motility and function are coupled and influence neuronal ageing. Mitochondrial transport and function are closely linked and we hypothesise that impaired motility leads to a loss of organellar functionality. Dysfunctional mitochondria, in turn, can affect their own motility, with a net result of locking the organelles into a vicious cycle of transport-function impairment. This is predicted to culminate in the accumulation of stalled and dysfunctional mitochondria, thus significantly contributing to the ageing process of neurons by failing to meet the functional demands of ageing cells.

of transport mechanistically affects neuronal ageing phenotypes. Mitochondria are the best characterised cargoes in transport studies during ageing and their transport and function are closely linked (Saxton et al., 2012). It is therefore conceivable that an early decrease in motility could trigger a rapid and dysfunctional response from the mitochondria ('transport to function' hypothesis, Figure 2). This may represent a causal event at the beginning of a damaging cascade leading to late onset neuronal dysfunction.

Mitochondria accumulate damage over time (Bratic and Larsson, 2013; Payne and Chinnery, 2015; Sun et al., 2016) and it has been suggested that mitochondrial dysfunction leads to transport reduction (Miller and Sheetz, 2004; Wang et al., 2011; Hwang et al., 2014; Grimm et al., 2018). Therefore, it is also possible that early mitochondrial damage could induce transport decline in early life ('function to transport' hypothesis, Figure 2). Reduced transport may, in turn, trigger a deleterious functional response in a vicious cycle that would ultimately lead to cellular dysfunction with increased age (Figure 2). Increased abundance of proteins involved in mitochondria function has been correlated to cognitive stability during human ageing and it would be interesting to understand whether these molecules impact on mitochondrial transport in later life (Wingo et al., 2019). Future studies should address in more detail the precise temporal cascade of events linking decreased motility to cellular dysfunction. Combining the knowledge derived from different model systems is predicted to facilitate our understanding of how mitochondrial transport is integrated within other cellular processes to affect ageing of neurons.

Gaining deeper insight into the mechanisms controlling age-dependent failure of cargo transport, including expanding the repertoire or cargoes imaged, might prove critical to understand how neuronal health declines in ageing organisms
(Table 1). Early impairments to regulatory nodes of intracellular trafficking could represent the initial hits of a multi-hit model of neurodegeneration (Sulzer, 2007; Garden and La Spada, 2012). In this view, it becomes a priority to identify early phenotypes at the beginning of a cascade of events leading to neuronal dysfunction. Recognising viable targets to restore cargo transport during ageing may therefore prove essential. Motor abundance (Puthanveettil et al., 2008; Li et al., 2016; Vagnoni and Bullock, 2018; Badal et al., 2019) and the activity of signalling pathways linked to lifespan extension (Li et al., 2016; Morsci et al., 2016; Vagnoni and Bullock, 2018) are potential candidates, even though these studies are limited to only few cargoes. More work is needed to understand the general mechanisms governing intracellular trafficking during ageing in a bid to target axonal transport, in conjunction with other cellular processes (Riera and Dillin, 2015), to improve the health of neurons during ageing (Figure 1).

\section{AUTHOR CONTRIBUTIONS}

AV wrote the initial draft of the manuscript with significant contribution from FM. AV and FM revised and approved the manuscript.

\section{FUNDING}

This work was funded through a NC3Rs David Sainsbury Fellowship and SKT grants (NC/N001753/2 and NC/T001224/1), a van Geest Ph.D. Studentship and a van Geest Fellowship in Dementia and Neurodegeneration from King's College London, a Royal Society Research Grant (RGS $\backslash R 2 \backslash 180450$ ), an Academy of Medical Sciences Springboard Award (SBF004\1088), and a BBSRC CTP Studentship (BB/T508597/1) to AV. 


\section{ACKNOWLEDGMENTS}

We would like to thank James Sleigh (UCL-IoN, United Kingdom) and Marina Ezcurra (University of Kent,

\section{REFERENCES}

Allen, R., Allen, N., and Travis, J. (1981). Video-enhanced contrast, differential interference contrast (AVEC-DIC) microscopy: a new method capable of analyzing microtubule-related motility in the reticulopodial network of Allogromia laticollaris. Cell Motil. 1, 291-302. doi: 10.1002/cm.970010303

Allen, R., Metuzals, J., Tasaki, I., Brady, S., and Gilbert, S. (1982). Fast axonal transport in squid giant axon. Science 218, 1127-1129. doi: 10.1126/science. 6183744

Avery, M. A., Rooney, T. M., Pandya, J. D., Wishart, T. M., Gillingwater, T. H., Geddes, J. W., et al. (2012). WldS prevents axon degeneration through increased mitochondrial flux and enhanced mitochondrial Ca2+ buffering. Curr. Biol. 22, 596-600. doi: 10.1016/j.cub.2012.02.043

Ayaz, D., Leyssen, M., Koch, M., Yan, J., Srahna, M., Sheeba, V., et al. (2008). Axonal injury and regeneration in the adult brain of Drosophila. J. Neurosci. 28, 6010-6021. doi: 10.1523/JNEUROSCI.0101-08.2008

Azpurua, J., Mahoney, R. E., and Eaton, B. A. (2018). Transcriptomics of aged Drosophila motor neurons reveals a matrix metalloproteinase that impairs motor function. Aging Cell 17:e12729. doi: 10.1111/acel.12729

Badal, K. K., Akhmedov, K., Lamoureux, P., Liu, X.-A., Reich, A., Fallahi-Sichani, M., et al. (2019). Synapse formation activates a transcriptional program for persistent enhancement in the bi-directional transport of mitochondria. Cell Rep. 26, 507.e3-517.e3. doi: 10.1016/j.celrep.2018.12.073

Baldwin, K. R., Godena, V. K., Hewitt, V. L., and Whitworth, A. J. (2016). Axonal transport defects are a common phenotype in Drosophila models of ALS. Hum. Mol. Genet. 25, 2378-2392. doi: 10.1093/hmg/ddw105

Bergamin, G., Cieri, D., Vazza, G., Argenton, F., and Mostacciuolo, M. L. (2016). Zebrafish $\operatorname{Tg}($ hb9:MTS-Kaede): a new in vivo tool for studying the axonal movement of mitochondria. Biochim. Biophys. Acta Gen. Subj. 1860, 1247-1255. doi: 10.1016/J.BBAGEN.2016.03.007

Bilsland, L. G., Sahai, E., Kelly, G., Golding, M., Greensmith, L., and Schiavo, G. (2010). Deficits in axonal transport precede ALS symptoms in vivo. Proc. Natl. Acad. Sci. U.S.A. 107, 20523-20528. doi: 10.1073/pnas. 1006869107

Bishop, N. A., Lu, T., and Yankner, B. A. (2010). Neural mechanisms of ageing and cognitive decline. Nature 464, 529-535. doi: 10.1038/nature08983

Bjedov, I., Toivonen, J. M., Kerr, F., Slack, C., Jacobson, J., Foley, A., et al. (2010). Mechanisms of life span extension by rapamycin in the fruit fly Drosophila melanogaster. Cell Metab. 11, 35-46. doi: 10.1016/J.CMET.2009.11.010

Bolea, I., Gan, W.-B., Manfedi, G., and Magrané, J. (2014). Imaging of mitochondrial dynamics in motor and sensory axons of living mice. Methods Enzymol. 547, 97-110. doi: 10.1016/B978-0-12-801415-8.00006-0

Brady, S. T., Lasek, R. J., and Allen, R. D. (1982). Fast axonal transport in extruded axoplasm from squid giant axon. Science 218, 1129-1131. doi: 10.1126/science. 6183745

Bratic, A., and Larsson, N.-G. (2013). The role of mitochondria in aging. J. Clin. Invest. 123, 951-957. doi: 10.1172/JCI64125

Breckwoldt, M. O., Pfister, F. M. J., Bradley, P. M., Marinković, P., Williams, P. R., Brill, M. S., et al. (2014). Multiparametric optical analysis of mitochondrial redox signals during neuronal physiology and pathology in vivo. Nat. Med. 20, 555-560. doi: 10.1038/nm.3520

Brunetti, M., Miscena, A., Salviati, A., and Gaiti, A. (1987). Effect of aging on the rate of axonal transport of choline-phosphoglycerides. Neurochem. Res. 12 61-65. doi: 10.1007/BF00971365

Cao, X., Wang, H., Wang, Z., Wang, Q., Zhang, S., Deng, Y., et al. (2017). In vivo imaging reveals mitophagy independence in the maintenance of axonal mitochondria during normal aging. Aging Cell 16, 1180-1190. doi: 10.1111/acel. 12654

Castel, M. N., Beaudet, A., and Laduron, P. M. (1994). Retrograde axonal transport of neurotensin in rat nigrostriatal dopaminergic neurons. Modulation during ageing and possible physiological role. Biochem. Pharmacol. 47, 53-62. doi: 10.1016/0006-2952(94)90437-5
United Kingdom) for critically reading the manuscript, Claire Fraser (University of Sidney, Australia) for suggestions with the human studies and the three reviewers for their comments on the manuscript.

Castelli, V., Benedetti, E., Antonosante, A., Catanesi, M., Pitari, G., Ippoliti, R., et al. (2019). Neuronal cells rearrangement during aging and neurodegenerative disease: metabolism, oxidative stress and organelles dynamic. Front. Mol. Neurosci. 12:132. doi: 10.3389/fnmol.2019.00132

Chang, D. T. W., Honick, A. S., and Reynolds, I. J. (2006). Mitochondrial trafficking to synapses in cultured primary cortical neurons. J. Neurosci. 26, 7035-7045. doi: 10.1523/JNEUROSCI.1012-06.2006

Clancy, D. J., Gems, D., Harshman, L. G., Oldham, S., Stocker, H., Hafen, E., et al. (2001). Extension of life-span by loss of CHICO, a Drosophila insulin receptor substrate protein. Science 292, 104-106. doi: 10.1126/science.1057991

Cross, D. J., Flexman, J. A., Anzai, Y., Maravilla, K. R., and Minoshima, S. (2008). Age-related decrease in axonal transport measured by MR imaging in vivo. Neuroimage 39, 915-926. doi: 10.1016/j.neuroimage.2007.08.036

Davalos, D., Lee, J. K., Smith, W. B., Brinkman, B., Ellisman, M. H., Zheng, B., et al. (2008). Stable in vivo imaging of densely populated glia, axons and blood vessels in the mouse spinal cord using two-photon microscopy. J. Neurosci. Methods 169, 1-7. doi: 10.1016/j.jneumeth.2007.11.011

De Vos, K. J., Grierson, A. J., Ackerley, S., and Miller, C. C. J. (2008). Role of axonal transport in neurodegenerative diseases. Annu. Rev. Neurosci. 31, 151-173. doi: 10.1146/annurev.neuro.31.061307.090711

Debattisti, V., Gerencser, A. A., Saotome, M., Das, S., and Hajnóczky, G. (2017). ROS control mitochondrial motility through p38 and the motor adaptor miro/trak. Cell Rep. 21, 1667-1680. doi: 10.1016/j.celrep.2017.10.060

Dey, S., Banker, G., and Ray, K. (2017). Anterograde transport of Rab4-associated vesicles regulates synapse organization in Drosophila. Cell Rep. 18, 2452-2463. doi: 10.1016/j.celrep.2017.02.034

DiLoreto, R., and Murphy, C. T. (2015). The cell biology of aging. Mol. Biol. Cell 26, 4524-4531. doi: 10.1091/mbc.E14-06-1084

Ding, L., Kuhne, W. W., Hinton, D. E., Song, J., and Dynan, W. S. (2010). Quantifiable biomarkers of normal aging in the japanese medaka fish (Oryzias latipes). PLoS One 5:e13287. doi: 10.1371/journal.pone.0013287

Dujardin, F. (1835). Recherches sur les organismes inférieurs III. Sur les prétendus estomacs des animalcules infusoires et sur une substance appelée sarcode. Ann. Sci. Nat. Zool. Biol. Anim. 4, 343-377.

Ezcurra, M., Benedetto, A., Sornda, T., Gilliat, A. F., Au, C., Zhang, Q., et al. (2018). C. elegans eats its own intestine to make yolk leading to multiple senescent pathologies. Curr. Biol. 28, 2544.e5-2556.e5. doi: 10.1016/J.CUB.2018.06.035

Faits, M., Zhang, C., Soto, F., and Kerschensteiner, D. (2016). Dendritic mitochondria reach stable positions during circuit development. eLife 5:e11583. doi: 10.7554/eLife.11583

Fanara, P., Wong, P.-Y. A., Husted, K. H., Liu, S., Liu, V. M., Kohlstaedt, L. A. et al. (2012). Cerebrospinal fluid-based kinetic biomarkers of axonal transport in monitoring neurodegeneration. J. Clin. Invest. 122, 3159-3169. doi: 10.1172/ JCI64575

Farrar, M. J., Bernstein, I. M., Schlafer, D. H., Cleland, T. A., Fetcho, J. R., and Schaffer, C. B. (2012). Chronic in vivo imaging in the mouse spinal cord using an implanted chamber. Nat. Methods 9, 297-302. doi: 10.1038/nmeth.1856

Fatouros, C., Pir, G. J., Biernat, J., Koushika, S. P., Mandelkow, E., Mandelkow, E.M., et al. (2012). Inhibition of tau aggregation in a novel Caenorhabditis elegans model of tauopathy mitigates proteotoxicity. Hum. Mol. Genet. 21, 3587-3603. doi: $10.1093 / \mathrm{hmg} / \mathrm{dds} 190$

Fernandez, H. L., and Hodges-Savola, C. A. (1994). Axoplasmic transport of calcitonin gene-related peptide in rat peripheral nerve as a function of age. Neurochem. Res. 19, 1369-1377. doi: 10.1007/BF00972465

Flurkey, K., Currer, J. M., and Harrison, D. E. (2007). "Mouse models in aging research," in The Mouse in Biomedical Research, 2nd Edn, Vol. 3, eds J. G. Fox, S. W. Barthold, M. T. Davisson, C. E. Newcomer, F. W. Quimby, and A. L. Smithcpesnm, (New York, NY: Elsevier), 637-672. doi: 10.1016/b978012369454-6/50074-1

Frolkis, V. V., Tanin, S. A., and Gorban, Y. N. (1997). Age-related changes in axonal transport. Exp. Gerontol. 32, 441-450. doi: 10.1016/S0531-5565(96)00168-4 
Garden, G. A., and La Spada, A. R. (2012). Intercellular (mis)communication in neurodegenerative disease. Neuron 73, 886-901. doi: 10.1016/j.neuron.2012. 02.017

Geinisman, Y., Bondareff, W., and Telser, A. (1977). Diminished axonal transport of glycoproteins in the senescent rat brain. Mech. Ageing Dev. 6, 363-378. doi: 10.1016/0047-6374(77)90038-0

Gems, D., and Riddle, D. L. (2000). Defining wild-type life span in Caenorhabditis elegans. J. Gerontol. Ser. A Biol. Sci. Med. Sci. 55, B215-B219. doi: 10.1093/ gerona/55.5.B215

Gerhard, G. S., Kauffman, E. J., Wang, X., Stewart, R., Moore, J. L., Kasales, C. J., et al. (2002). Life spans and senescent phenotypes in two strains of Zebrafish (Danio rerio). Exp. Gerontol. 37, 1055-1068. doi: 10.1016/S05315565(02)00088-8

Gibbs, K. L., Kalmar, B., Sleigh, J. N., Greensmith, L., and Schiavo, G. (2016). In vivo imaging of axonal transport in murine motor and sensory neurons. J. Neurosci. Methods 257, 26-33. doi: 10.1016/j.jneumeth.2015.09.018

Gilley, J., Seereeram, A., Ando, K., Mosely, S., Andrews, S., Kerschensteiner, M., et al. (2012). Age-dependent axonal transport and locomotor changes and tau hypophosphorylation in a "P301L" tau knockin mouse. Neurobiol. Aging 33, 621.e1-621.e15. doi: 10.1016/j.neurobiolaging.2011.02.014

Gray, D. C., Wolfe, R., Gee, B. P., Scoles, D., Geng, Y., Masella, B. D., et al. (2008). In Vivo imaging of the fine structure of rhodamine-labeled macaque retinal ganglion cells. Invest. Opthalmol. Vis. Sci. 49, 467-473. doi: 10.1167/iovs.070605

Grimm, A., Cummins, N., and Götz, J. (2018). Local oxidative damage in the soma and dendrites quarantines neuronal mitochondria at the site of insult. iScience 6, 114-127. doi: 10.1016/j.isci.2018.07.015

Gunawardena, S., and Goldstein, L. S. (2001). Disruption of axonal transport and neuronal viability by amyloid precursor protein mutations in Drosophila. Neuron 32, 389-401. doi: 10.1016/S0896-6273(01)00496-2

Guo, X., Macleod, G. T., Wellington, A., Hu, F., Panchumarthi, S., Schoenfield, M., et al. (2005). The GTPase dMiro is required for axonal transport of mitochondria to Drosophila synapses. Neuron 47, 379-393. doi: 10.1016/j. neuron.2005.06.027

Gutnick, A., Banghart, M. R., West, E. R., and Schwarz, T. L. (2019). The lightsensitive dimerizer zapalog reveals distinct modes of immobilization for axonal mitochondria. Nat. Cell Biol. 21, 768-777. doi: 10.1038/s41556-019-0317-2

Hall, A. R. (1965). Galileo and the science of motion. Br. J. Hist. Sci. 2, 185-199. doi: 10.1017/S0007087400002193

Harel, I., Benayoun, B. A., Machado, B., Singh, P. P., Hu, C.-K., Pech, M. F., et al. (2015). A platform for rapid exploration of aging and diseases in a naturally short-lived vertebrate. Cell 160, 1013-1026. doi: 10.1016/j.cell.2015.01.038

Hollenbeck, P. J., and Saxton, W. M. (2005). The axonal transport of mitochondria. J. Cell Sci. 118, 5411-5419. doi: 10.1242/jcs.02745

Huxley, T. H. (ed.) (1869). On the Physical Basis of Life. New Haven, CT: The College Courant.

Hwang, R.-D., Wiemerslage, L., LaBreck, C. J., Khan, M., Kannan, K., Wang, X., et al. (2014). The neuroprotective effect of human uncoupling protein 2 (hUCP2) requires cAMP-dependent protein kinase in a toxin model of Parkinson's disease. Neurobiol. Dis. 69, 180-191. doi: 10.1016/j.nbd.2014. 05.032

Kenyon, C., Chang, J., Gensch, E., Rudner, A., and Tabtiang, R. (1993). A C. elegans mutant that lives twice as long as wild type. Nature 366, 461-464. doi: 10.1038/ $366461 \mathrm{a} 0$

Kerschensteiner, M., Schwab, M. E., Lichtman, J. W., and Misgeld, T. (2005). In vivo imaging of axonal degeneration and regeneration in the injured spinal cord. Nat. Med. 11, 572-577. doi: 10.1038/nm1229

Kim, Y., Zheng, X., Ansari, Z., Bunnell, M. C., Herdy, J. R., Traxler, L., et al. (2018). Mitochondrial aging defects emerge in directly reprogrammed human neurons due to their metabolic profile. Cell Rep. 23, 2550-2558. doi: 10.1016/j.celrep. 2018.04.105

Klass, M., Nguyen, P. N., and Dechavigny, A. (1983). Age-correlated changes in the DNA template in the nematode Caenorhabditis elegans. Mech. Ageing Dev. 22, 253-263. doi: 10.1016/0047-6374(83)90080-5

Kudumala, S. R., Penserga, T., Börner, J., Slipchuk, O., Kakad, P., Lee, L. H., et al. (2017). Lissencephaly-1 dependent axonal retrograde transport of L1-type CAM Neuroglian in the adult drosophila central nervous system. PLoS One 12:e0183605. doi: 10.1371/journal.pone.0183605
Kosman, A. L. (1969). Aristotle's definition of motion. Phronesis 14, 40-62. doi: $10.1163 / 156852869$ X00037

Lane, N. (2015). The unseen world: reflections on Leeuwenhoek (1677) 'Concerning little animals'. Philos. Trans. R. Soc. B Biol. Sci. 370, 20140344. doi: 10.1098/RSTB.2014.0344

Lasek, R. J. (1967). Bidirectional transport of radioactively labelled axoplasmic components. Nature 216, 1212-1214. doi: 10.1038/2161212a0

Lewis, T. L., Turi, G. F., Kwon, S.-K., Losonczy, A., and Polleux, F. (2016). Progressive decrease of mitochondrial motility during maturation of cortical axons in vitro and in vivo. Curr. Biol. 26, 2602-2608. doi: 10.1016/j.cub.2016. 07.064

Li, L. B., Lei, H., Arey, R. N., Li, P., Liu, J., Murphy, C. T., et al. (2016). The neuronal kinesin UNC-104/KIF1A is a key regulator of synaptic aging and insulin signaling-regulated memory. Curr. Biol. 26, 605-615. doi: 10.1016/j.cub. 2015.12.068

Li, W., Hoffman, P. N., Stirling, W., Price, D. L., and Lee, M. K. (2004). Axonal transport of human alpha-synuclein slows with aging but is not affected by familial Parkinson's disease-linked mutations. J. Neurochem. 88, 401-410. doi: 10.1046/j.1471-4159.2003.02166.x

Lichtman, J. W., and Fraser, S. E. (2001). The neuronal naturalist: watching neurons in their native habitat. Nat. Neurosci. 4, 1215-1220. doi: 10.1038/nn754

Lin, M.-Y., Cheng, X.-T., Tammineni, P., Xie, Y., Zhou, B., Cai, Q., et al. (2017). Releasing syntaphilin removes stressed mitochondria from axons independent of mitophagy under pathophysiological conditions. Neuron 94, 595.e6-610.e6. doi: 10.1016/j.neuron.2017.04.004

Linford, N. J., Bilgir, C., Ro, J., and Pletcher, S. D. (2013). Measurement of lifespan in Drosophila melanogaster. J. Vis. Exp. 7:50068. doi: 10.3791/50068

Liu, G.-H., Barkho, B. Z., Ruiz, S., Diep, D., Qu, J., Yang, S.-L., et al. (2011). Recapitulation of premature ageing with iPSCs from Hutchinson-Gilford progeria syndrome. Nature 472, 221-225. doi: 10.1038/nature09879

Liu, Z., Kurokawa, K., Zhang, F., Lee, J. J., and Miller, D. T. (2017). Imaging and quantifying ganglion cells and other transparent neurons in the living human retina. Proc. Natl. Acad. Sci. U.S.A. 114, 12803-12808. doi: 10.1073/pnas. 1711734114

López-Lluch, G., Irusta, P. M., Navas, P., and de Cabo, R. (2008). Mitochondrial biogenesis and healthy aging. Exp. Gerontol. 43, 813-819. doi: 10.1016/j.exger. 2008.06.014

López-Otín, C., Blasco, M. A., Partridge, L., Serrano, M., and Kroemer, G. (2013). The hallmarks of aging. Cell 153, 1194-1217. doi: 10.1016/j.cell.2013.05.039

Lubinska, L. (1964). Axoplasmic streaming in regenerating and in normal nerve fibres. Prog. Brain Res. 13, 1-71. doi: 10.1016/S0079-6123(08)60139-9

Maday, S., and Holzbaur, E. L. F. (2014). Autophagosome biogenesis in primary neurons follows an ordered and spatially regulated pathway. Dev. Cell 30, 71-85. doi: 10.1016/j.devcel.2014.06.001

Mandal, A., Pinter, K., and Drerup, C. M. (2018). Analyzing neuronal mitochondria in vivo using fluorescent reporters in zebrafish. Front. Cell Dev. Biol. 6:144. doi: 10.3389/fcell.2018.00144

Mar, F. M., Simoes, A. R., Leite, S., Morgado, M. M., Santos, T. E., Rodrigo, I. S., et al. (2014). CNS axons globally increase axonal transport after peripheral conditioning. J. Neurosci. 34, 5965-5970. doi: 10.1523/JNEUROSCI.4680-13. 2014

McQuarrie, I. G., Brady, S. T., and Lasek, R. J. (1989). Retardation in the slow axonal transport of cytoskeletal elements during maturation and aging. Neurobiol. Aging 10, 359-365. doi: 10.1016/0197-4580(89)90049-3

Medioni, C., Ephrussi, A., and Besse, F. (2015). Live imaging of axonal transport in Drosophila pupal brain explants. Nat. Protoc. 10, 574-584. doi: 10.1038/nprot. 2015.034

Mellone, M., Kestoras, D., Andrews, M. R., Dassie, E., Crowther, R. A., Stokin, G. B., et al. (2013). Tau pathology is present in vivo and develops in vitro in sensory neurons from human P301S tau transgenic mice: a system for screening drugs against tauopathies. J. Neurosci. 33, 18175-18189. doi: 10.1523/JNEUROSCI. 4933- 12.2013

Mertens, J., Paquola, A. C. M., Ku, M., Hatch, E., Böhnke, L., Ladjevardi, S., et al. (2015). Directly reprogrammed human neurons retain aging-associated transcriptomic signatures and reveal age-related nucleocytoplasmic defects. Cell Stem Cell 17, 705-718. doi: 10.1016/j.stem.2015.09.001

Milde, S., Adalbert, R., Elaman, M. H., and Coleman, M. P. (2015). Axonal transport declines with age in two distinct phases separated by a period of 
relative stability. Neurobiol. Aging 36, 971-981. doi: 10.1016/j.neurobiolaging. 2014.09.018

Millecamps, S., and Julien, J.-P. (2013). Axonal transport deficits and neurodegenerative diseases. Nat. Rev. Neurosci. 14, 161-176. doi: 10.1038/nrn3380

Miller, K. E., and Sheetz, M. P. (2004). Axonal mitochondrial transport and potential are correlated. J. Cell Sci. 117, 2791-2804. doi: 10.1242/jcs.01130

Misgeld, T., Kerschensteiner, M., Bareyre, F. M., Burgess, R. W., and Lichtman, J. W. (2007). Imaging axonal transport of mitochondria in vivo. Nat. Methods 4, 559-561. doi: 10.1038/nmeth1055

Misgeld, T., and Schwarz, T. L. (2017). Mitostasis in neurons: maintaining mitochondria in an extended cellular architecture. Neuron 96, 651-666. doi: 10.1016/j.neuron.2017.09.055

Mondal, S., Ahlawat, S., Rau, K., Venkataraman, V., and Koushika, S. P. (2011). Imaging in vivo neuronal transport in genetic model organisms using microfluidic devices. Traffic 12, 372-385. doi: 10.1111/j.1600-0854.2010. 01157.x

Morris, J. Z., Tissenbaum, H. A., and Ruvkun, G. (1996). A phosphatidylinositol-3$\mathrm{OH}$ kinase family member regulating longevity and diapause in Caenorhabditis elegans. Nature 382, 536-539. doi: 10.1038/382536a0

Morsci, N. S., Hall, D. H., Driscoll, M., and Sheng, Z.-H. (2016). Agerelated phasic patterns of mitochondrial maintenance in adult Caenorhabditis elegans neurons. J Neurosci 36, 1373-1385. doi: 10.1523/JNEUROSCI.2799-15. 2016

Niccoli, T., and Partridge, L. (2012). Ageing as a risk factor for disease. Curr. Biol. 22, R741-R752. doi: 10.1016/j.cub.2012.07.024

Nikoletopoulou, V., and Tavernarakis, N. (2012). Calcium homeostasis in aging neurons. Front. Genet. 3:200. doi: 10.3389/fgene.2012.00200

Obashi, K., and Okabe, S. (2013). Regulation of mitochondrial dynamics and distribution by synapse position and neuronal activity in the axon. Eur. J. Neurosci. 38, 2350-2363. doi: 10.1111/ejn.12263

Ochs, S. (1973). Effect of maturation and aging on the rate of fast axoplasmic transport in mammalian nerve. Prog. Brain Res. 40, 349-362. doi: 10.1016/ S0079-6123(08)60699-8

O’Donnell, K. C., Vargas, M. E., and Sagasti, A. (2013). WldS and PGC-1 regulate mitochondrial transport and oxidation state after axonal injury. J. Neurosci. 33, 14778-14790. doi: 10.1523/JNEUROSCI.1331-13.2013

Parihar, M. S., and Brewer, G. J. (2007). Simultaneous age-related depolarization of mitochondrial membrane potential and increased mitochondrial reactive oxygen species production correlate with age-related glutamate excitotoxicity in rat hippocampal neurons. J. Neurosci. Res. 85, 1018-1032. doi: 10.1002/jnr. 21218

Pathak, D., Sepp, K. J., and Hollenbeck, P. J. (2010). Evidence that myosin activity opposes microtubule-based axonal transport of mitochondria. J. Neurosci. 30, 8984-8992. doi: 10.1523/JNEUROSCI.1621-10.2010

Patten, S. A., Armstrong, G. A. B., Lissouba, A., Kabashi, E., Parker, J. A., and Drapeau, P. (2014). Fishing for causes and cures of motor neuron disorders. Dis. Model. Mech. 7, 799-809. doi: 10.1242/dmm.015719

Payne, B. A. I., and Chinnery, P. F. (2015). Mitochondrial dysfunction in aging: much progress but many unresolved questions. Biochim. Biophys. Acta 1847, 1347-1353. doi: 10.1016/j.bbabio.2015.05.022

Pilling, A. D., Horiuchi, D., Lively, C. M., and Saxton, W. M. (2006). Kinesin-1 and dynein are the primary motors for fast transport of mitochondria in Drosophila motor axons. Mol. Biol. Cell 17, 2057-2068. doi: 10.1091/mbc.E05-06-0526

Plucinska, G., Paquet, D., Hruscha, A., Godinho, L., Haass, C., Schmid, B., et al. (2012). In Vivo imaging of disease-related mitochondrial dynamics. J. Neurosci. 32, 16203-16212. doi: 10.1523/JNEUROSCI.1327-12.2012

Puthanveettil, S. V., Monje, F. J., Miniaci, M. C., Choi, Y.-B., Karl, K. A., Khandros, E., et al. (2008). A new component in synaptic plasticity: upregulation of kinesin in the neurons of the gill-withdrawal reflex. Cell 135, 960-973. doi: 10.1016/j. cell.2008.11.003

Quraishe, S., Cowan, C. M., and Mudher, A. (2013). NAP (davunetide) rescues neuronal dysfunction in a Drosophila model of tauopathy. Mol. Psychiatry 18, 834-842. doi: 10.1038/mp.2013.32

Rawson, R. L., Yam, L., Weimer, R. M., Bend, E. G., Hartwieg, E., Horvitz, H. R., et al. (2014). Axons degenerate in the absence of mitochondria in C. elegans. Curr. Biol. 24, 760-765. doi: 10.1016/j.cub.2014.02.025

Reis, G. F., Yang, G., Szpankowski, L., Weaver, C., Shah, S. B., Robinson, J. T., et al. (2012). Molecular motor function in axonal transport in vivo probed by genetic and computational analysis in Drosophila. Mol. Biol. Cell 23, 1700-1714. doi: 10.1091/mbc.E11-11-0938

Riera, C. E., and Dillin, A. (2015). Can aging be "drugged" Nat. Med. 21, 1400-1405. doi: $10.1038 / \mathrm{nm} .4005$

Sajic, M., Mastrolia, V., Lee, C. Y., Trigo, D., Sadeghian, M., Mosley, A. J., et al. (2013). Impulse conduction increases mitochondrial transport in adult mammalian peripheral nerves in vivo. PLoS Biol. 11:e1001754. doi: 10.1371/ journal.pbio.1001754

Samuels, A. J., Boyarsky, L. L., Gerard, R. W., Libet, B., and Brust, M. (1951). Distribution exchange and migration of phosphate compounds in the nervous system. Am. J. Physiol. 164, 1-15. doi: 10.1152/ajplegacy.1950.164.1.1

Saxton, W. M., Hollenbeck, P. J., Griffiths, G., and Burkhardt, J. K. (2012). The axonal transport of mitochondria. J. Cell Sci. 125, 2095-2104. doi: 10.1242/jcs. 053850

Schmieg, N., Menendez, G., Schiavo, G., and Terenzio, M. (2014). Signalling endosomes in axonal transport: travel updates on the molecular highway. Semin. Cell Dev. Biol. 27, 32-43. doi: 10.1016/J.SEMCDB.2013.10.004

Seo, A. Y., Joseph, A.-M., Dutta, D., Hwang, J. C. Y., Aris, J. P., and Leeuwenburgh, C. (2010). New insights into the role of mitochondria in aging: mitochondrial dynamics and more. J. Cell Sci. 123, 2533-2542. doi: 10.1242/jcs.070490

Sheng, Z.-H., and Cai, Q. (2012). Mitochondrial transport in neurons: impact on synaptic homeostasis and neurodegeneration. Nat. Rev. Neurosci. 13, 77-93. doi: $10.1038 / \mathrm{nrn} 3156$

Siekevitz, P. (1957). Powerhouse of the cell. Sci. Am. 197, 131-144. doi: 10.2307/ 24940890

Sleigh, J., and Schiavo, G. (2016). Older but not slower: aging does not alter axonal transport dynamics of signalling endosome in vivo. Matters. doi: 10.19185/ matters.201605000018

Sleigh, J. N., Tosolini, A. P., Gordon, D., Devoy, A., Fratta, P., Fisher, E. M. C., et al. (2018). ALS mice carrying pathological mutant TDP-43, but not mutant FUS, display axonal transport defects in vivo. bioRxiv

Sleigh, J. N., Vagnoni, A., Twelvetrees, A. E., and Schiavo, G. (2017). Methodological advances in imaging intravital axonal transport. F1000Research 6:200. doi: 10.12688/f1000research.10433.1

Smith, G. A., Lin, T.-H., Sheehan, A. E., Van der Goes van Naters, W., Neukomm, L. J., Graves, H. K., et al. (2019). Glutathione S-Transferase regulates mitochondrial populations in axons through increased glutathione oxidation. Neuron 103, 52.e6-65.e6. doi: 10.1016/j.neuron.2019.04.017

Smit-rigter, L., Rajendran, R., Silva, C. A. P., Spierenburg, L., Groeneweg, F., Ruimschotel, E. M., et al. (2016). Mitochondrial dynamics in visual cortex are limited in vivo and not affected by axonal structural report mitochondrial dynamics in visual cortex are limited in vivo and not affected by axonal structural plasticity. Curr. Biol. 26, 2609-2616. doi: 10.1016/j.cub.2016.07.033

Sorbara, C. D., Wagner, N. E., Ladwig, A., Nikić, I., Merkler, D., Kleele, T., et al. (2014). Pervasive axonal transport deficits in multiple sclerosis models. Neuron 84, 1183-1190. doi: 10.1016/j.neuron.2014.11.006

Stavoe, A. K., Gopal, P. P., Gubas, A., Tooze, S. A., and Holzbaur, E. L. (2019). Expression of WIPI2B counteracts age-related decline in autophagosome biogenesis in neurons. eLife 8:e44219. doi: 10.7554/eLife.44219

Stromska, D. P., and Ochs, S. (1982). Axoplasmic transport in aged rats. Exp. Neurol. 77, 215-224. doi: 10.1016/0014-4886(82)90155-8

Sulzer, D. (2007). Multiple hit hypotheses for dopamine neuron loss in Parkinson's disease. Trends Neurosci. 30, 244-250. doi: 10.1016/j.tins.2007.03.009

Sun, N., Youle, R. J., and Finkel, T. (2016). The mitochondrial basis of aging. Mol. Cell 61, 654-666. doi: 10.1016/j.molcel.2016.01.028

Takihara, Y., Inatani, M., Eto, K., Inoue, T., Kreymerman, A., Miyake, S., et al. (2015). In vivo imaging of axonal transport of mitochondria in the diseased and aged mammalian CNS. Proc. Natl. Acad. Sci. U.S.A. 112, 10515-10520. doi: $10.1073 /$ pnas. 1509879112

Uchida, A., Komiya, Y., Tashiro, T., Yorifuji, H., Kishimoto, T., Nabeshima, Y., et al. (2001). Neurofilaments of Klotho, the mutant mouse prematurely displaying symptoms resembling human aging. J. Neurosci. Res. 64, 364-370. doi: 10.1002/ jnr.1087

UN DESA (2017). World Population World Population Prospects: The 2017 Revision, Key Findings and Advance Tables. Working Paper No. ESA/P/WP/248.

Vagnoni, A., and Bullock, S. L. (2016). A simple method for imaging axonal transport in aging neurons using the adult Drosophila wing. Nat. Protoc. 11, 1711-1723. doi: 10.1038 /nprot.2016.112 
Vagnoni, A., and Bullock, S. L. (2018). A cAMP/PKA/Kinesin-1 axis promotes the axonal transport of mitochondria in aging Drosophila neurons. Curr. Biol. 28, 1265.e4-1272.e4. doi: 10.1016/j.cub.2018.02.048

Vagnoni, A., Hoffmann, P. C., and Bullock, S. L. (2016). Reducing Lissencephaly1 levels augments mitochondrial transport and has a protective effect in adult Drosophila neurons. J. Cell Sci. 129, 178-190. doi: 10.1242/jcs.17 9184

Viancour, T. A., and Kreiter, N. A. (1993). Vesicular fast axonal transport rates in young and old rat axons. Brain Res. 628, 209-217. doi: 10.1016/0006-8993(93) 90957-O

Wakamatsu, Y., Pristyazhnyuk, S., Kinoshita, M., Tanaka, M., and Ozato, K. (2001). The see-through medaka: a fish model that is transparent throughout life. Proc. Natl. Acad. Sci. U.S.A. 98, 10046-10050. doi: 10.1073/pnas.18120 4298

Walker, A. S., Burrone, J., and Meyer, M. P. (2013). Functional imaging in the zebrafish retinotectal system using RGECO. Front. Neural Circuits 7:34. doi: 10.3389/fncir.2013.00034

Wang, X., and Schwarz, T. L. (2009). The mechanism of Ca2+-dependent regulation of kinesin-mediated mitochondrial motility. Cell 136, 163-174. doi: 10.1016/j.cell.2008.11.046

Wang, X., Winter, D., Ashrafi, G., Schlehe, J., Wong, Y. L., Selkoe, D., et al. (2011). PINK1 and Parkin target Miro for phosphorylation and degradation to arrest mitochondrial motility. Cell 147, 893-906. doi: 10.1016/j.cell.2011.10.018

Weiss, P. (1967). Neuronal dynamics and axonal flow. 3. Cellulifugal transport of labeled neuroplasm in isolated nerve preparations. Proc. Natl. Acad. Sci. U.S.A. 57, 1239-1245. doi: 10.1073/pnas.57.5.1239

Weiss, P., Wang, H., Taylor, A. C., and Edds, M. V. (1945). Proximo-distal fluid convection in the endoneurial spaces of peripheral nerves, demonstrated by colored and radioactive (isotope) tracers. Am. J. Physiol. Content 143, 521-540. doi: 10.1152/ajplegacy.1945.143.4.521
Wingo, A. P., Dammer, E. B., Breen, M. S., Logsdon, B. A., Duong, D. M., Troncosco, J. C., et al. (2019). Large-scale proteomic analysis of human brain identifies proteins associated with cognitive trajectory in advanced age. Nat. Commun. 10:1619. doi: 10.1038/s41467-019-09613-z

Xiong, X., Wang, X., Ewanek, R., Bhat, P., DiAntonio, A., and Collins, C. A. (2010). Protein turnover of the Wallenda/DLK kinase regulates a retrograde response to axonal injury. J. Cell Biol. 191, 211-223. doi: 10.1083/jcb.201006039

$\mathrm{Xu}, \mathrm{Y}$. , Chen, M., Hu, B., Huang, R., and Hu, B. (2017). In vivo imaging of mitochondrial transport in single-axon regeneration of zebrafish mauthner cells. Front. Cell. Neurosci. 11:4. doi: 10.3389/fncel.2017.00004

Zhou, B., Yu, P., Lin, M. Y., Sun, T., Chen, Y., and Sheng, Z. H. (2016). Facilitation of axon regeneration by enhancing mitochondrial transport and rescuing energy deficits. J. Cell Biol. 214, 103-119. doi: 10.1083/jcb.201605101

Zhu, Y.-B., and Sheng, Z.-H. (2011). Increased axonal mitochondrial mobility does not slow amyotrophic lateral sclerosis (ALS)-like disease in mutant SOD1 mice. J. Biol. Chem. 286, 23432-23440. doi: 10.1074/jbc.M111.237818

Ziff, O. J., and Patani, R. (2019). Harnessing cellular aging in human stem cell models of amyotrophic lateral sclerosis. Aging Cell 18:e12862. doi: 10.1111/acel. 12862

Conflict of Interest Statement: The authors declare that the research was conducted in the absence of any commercial or financial relationships that could be construed as a potential conflict of interest.

Copyright (C) 2019 Mattedi and Vagnoni. This is an open-access article distributed under the terms of the Creative Commons Attribution License (CC BY). The use, distribution or reproduction in other forums is permitted, provided the original author(s) and the copyright owner(s) are credited and that the original publication in this journal is cited, in accordance with accepted academic practice. No use, distribution or reproduction is permitted which does not comply with these terms. 